\title{
A SUFFICIENT CONDITION FOR HYPERINVARIANCE
}

\author{
W. E. LONGSTAFF
}

\begin{abstract}
A linear transformation on a finite-dimensional complex linear space has the property that all of its invariant subspaces are hyperinvariant if and only if its lattice of invariant subspaces is distributive [1]. It is shown that an operator on a complex Hilbert space has this property if its lattice of invariant subspaces satisfies a certain distributivity condition.
\end{abstract}

1. Preliminaries. Throughout this paper $H$ will denote an arbitrary complex Hilbert space. All operators are bounded and all subspaces are closed. By a subspace lattice on $H$ is meant a family of subspaces of $H$ which is closed under the formation of arbitrary intersections and arbitrary closed linear spans and which contains the zero subspace $(0)$ and $H$. The family of subspaces invariant under an operator $T$ is denoted by Lat $T$. This is a subspace lattice as is the family of subspaces invariant under every operator commuting with $T$ which we denote by Hyperlat $T$. The elements of Hyperlat $T$ are called the hyperinvariant subspaces of $T$. Clearly Hyperlat $T \subseteq$ Lat $T$. A subspace lattice $\mathscr{F}$ is called commutative if for every pair of subspaces $M$, $N \in \widetilde{F}^{*}$ the corresponding projections $P_{M}$ and $P_{N}$ commute. Let $L$ be an abstract lattice. We say that $L$ is

(i) distributive if

$$
a \vee(b \wedge c)=(a \vee b) \wedge(a \vee c) \quad(a, b, c \in L)
$$

and its dual statement holds identically;

(ii) $\sigma$-infinitely meet distributive if $L$ is $\sigma$-complete and

$$
a \vee\left\{\bigwedge b_{n}: n \geqslant 1\right\}=\bigwedge\left\{a \vee b_{n}: n \geqslant 1\right\} \quad\left(a, b_{n} \in L\right)
$$

holds identically in $L$.

That the dual equation defining distributivity are equivalent to each other is an elementary result of lattice theory.

2. A sufficient condition for hyperinvariance. The key to the sufficient condition is a result of Sarason and the following lattice-theoretic result.

Proposition 2.1. If $L$ is an abstract $\sigma$-infinitely meet distributive lattice and $\theta: L \rightarrow L$ is a lattice automorphism with the properties

(I) $a \leqslant \theta(a) \vee \theta^{-1}(a)(a \in L)$;

(II) $a, \theta(a)$ comparable implies $a=\theta(a)$,

then $\theta$ is the identity automorphism.

Proof. For every $n \geqslant 1$ let $\theta^{n}: L \rightarrow L$ be defined in the obvious way. Let $a$

Received by the editors March 27, 1975.

AMS (MOS) subject classifications (1970). Primary 47A15; Secondary 26A30.

Copyright 1977, American Mathematical Society 
be an arbitrary fixed element of $L$. For $n \geqslant 1$ put $a_{n}=a \wedge \theta(a) \wedge$ $\theta^{2}(a) \wedge \cdots \wedge \theta^{n}(a)$. Then $a_{n} \wedge \theta\left(a_{n}\right)=a_{n+1}(n \geqslant 1)$. Using (I) and the fact that $L$ is distributive the statement

$$
\theta(x)=(x \wedge \theta(x)) \vee[\theta(x \wedge \theta(x))]
$$

holds identically in $L$. Using this identity it is easily shown that $\theta(a)=a_{1} \vee$ $\theta\left(a_{n}\right)(n \geqslant 1)$. Thus

$$
\theta(a)=\bigwedge\left\{a_{1} \vee \theta\left(a_{n}\right): n \geqslant 1\right\}=a_{1} \vee\left(\bigwedge\left\{\theta\left(a_{n}\right): n \geqslant 1\right\}\right)
$$

If $c=\bigwedge\left\{a_{n}: n \geqslant 1\right\}$ then

$$
\theta(c)=\bigwedge\left\{\theta\left(a_{n}\right): n \geqslant 1\right\} \geqslant \bigwedge\left\{a_{n+1}: n \geqslant 1\right\} \geqslant c
$$

and by (II), $\theta(c)=c$. Hence $\theta(a)=a_{1} \vee \theta(c)=a_{1} \vee c=a_{1} \leqslant a$ and again by (II), $\theta(a)=a$. This completes the proof.

Let $T$ be an operator on $H$. Notice that if $S$ is an invertible operator commuting with $T$ then $S M \in$ Lat $T$ ( $M \in$ Lat $T$ ) and the mapping $M \rightarrow S M$ is a lattice automorphism with the mapping $M \rightarrow S^{-1} M$ as its inverse. If the operator $A$ commutes with $T$ and $\mu$ is a scalar with $|\mu|>\|A\|$, the operator $S=A-\mu$ is invertible, commutes with $T$ and Lat $A=$ Lat $S$. By a result of Sarason [4], Lat $S=$ Lat $S^{-1}$. It readily follows that Hyperlat $T$ = Lat $T$ if and only if for every invertible operator $S$ commuting with $T$ satisfying Lat $S=$ Lat $S^{-1}$ the mapping $M \rightarrow S M(M \in$ Lat $T)$ is the identity automorphism.

Proposition 2.2. If Lat $T$ is distributive and $S$ is an invertible operator commuting with $T$, then $M \subseteq S M \vee S^{-1} M(M \in$ Lat $T)$.

Proof. Choose $\lambda$ with $0<\lambda<1 /\|S\|$. The operator $C=1+\lambda S$ is invertible and commutes with $T$. Let $M \in$ Lat $T$. It is readily verified that $C M \cap S M=C(M \cap S M)$ and $C M \cap M=C\left(M \cap S^{-1} M\right)$. Since $C M$ $\subseteq S M \vee M$, by distributivity we have

$$
\begin{aligned}
C M & =(C M \cap S M) \vee(C M \cap M)=C(M \cap S M) \vee C\left(M \cap S^{-1} M\right) \\
& =C\left(M \cap\left[S M \vee S^{-1} M\right]\right)
\end{aligned}
$$

and the result follows.

TheOrem 2.3. If Lat $T$ is $\sigma$-infinitely meet distributive Hyperlat $T=$ Lat $T$.

Proof. By our earlier remarks it suffices to show that if $S$ is an invertible operator commuting with $T$ and satisfying Lat $S=$ Lat $S^{-1}$ then the automorphism $M \rightarrow S M$ of Lat $T$ is the identity automorphism. Since Lat $T$ is distributive, this automorphism satisfies condition (I) of Proposition 2.1 by Proposition 2.2. Since Lat $S=$ Lat $S^{-1}$, condition (II) is also satisfied. The result now follows from Proposition 2.1.

Corollary 2.3.1. Hyperlat $\mathrm{T}=$ Lat $\mathrm{T}$ if Lat $\mathrm{T}$ is any one of the following:

(i) commutative;

(ii) isomorphic to the direct product of complete chains;

(iii) totally ordered. 
Proof. It is clear that in cases (ii) and (iii) Lat $T$ is $\sigma$-infinitely meet distributive. Suppose that Lat $T$ is commutative. Then it is also distributive. This follows from the fact that if $P$ and $Q$ are commuting projections then $P Q$ is the projection onto the intersection of the ranges of $P$ and $Q$ and $P+Q-P Q$ is the projection onto the closed linear span of the ranges of $P$ and $Q$. Thus if $K, L, M \in$ Lat $T$ then

$$
\begin{aligned}
P_{K \cap(L \vee M)} & =P_{K} P_{L \vee M}=P_{K}\left(P_{L}+P_{M}-P_{L} P_{M}\right) \\
& =P_{K \cap L}+P_{K \cap M}-P_{K \cap L} P_{K \cap M}=P_{(K \cap L) \vee(K \cap M)} .
\end{aligned}
$$

Now let

$$
K, L_{n}(n \geqslant 1) \in \text { Lat } T \text {. }
$$

Then $P_{\cap\left\{L_{n}: n \geqslant 1\right\}}$, respectively $P_{\cap\left\{K \vee L_{n}: n \geqslant 1\right\}}$, is the strong limit of the sequence $\left\{P_{\cap\left\{L_{n}: 1 \leqslant n \leqslant k\right\}}: k \geqslant 1\right\}$, respectively $\left\{P_{\cap\left\{K \vee L_{n}: 1 \leqslant n \leqslant k\right\}}: k \geqslant 1\right\}$. But

$$
\begin{aligned}
P_{\cap\left\{K \vee L_{n}: 1<n<k\right\}} & =P_{K \vee \cap\left\{L_{n}: 1<n<k\right\}} \\
& =P_{K}+P_{\cap\left\{L_{n}: 1<n<k\right\}}-P_{K} P_{\cap\left\{L_{n}: 1<n<k\right\}} .
\end{aligned}
$$

Taking strong limits gives

$$
P_{\cap\left\{K \vee L_{n}: n>1\right\}}=P_{K}+P_{\cap\left\{L_{n}: n>1\right\}}-P_{K} P_{\cap\left\{L_{n}: n>1\right\}}=P_{K \vee \cap\left\{L_{n}: n>1\right\}}
$$

Hence Lat $T$ is $\sigma$-infinitely meet distributive. The result follows by applying Theorem 2.3.

The results (i) and (iii) above are not new. The former was proved in [2] and the latter in [3].

3. Concluding remarks. It is a simple exercise to show for any linear transformation $T$ on a finite-dimensional complex linear space that Hyperlat $T$ is distributive and finite, therefore $\sigma$-infinitely meet distributive. It seems an interesting question whether Hyperlat $T$ is always $\sigma$-infinitely meet distributive or even whether the converse of Theorem 2.3 holds. For a normal operator $T$, Hyperlat $T$ consists of the ranges of the spectral projections for $T$ [2] and so is both commutative and a Boolean algebra and so is certainly $\sigma$-infinitely meet distributive.

\section{REFERENCES}

1. L. Brickman and P. A. Fillmore, The invariant subspace lattice of a linear transformation, Canad. J. Math. 19(1967), 810-822. MR 35 \#4242.

2. R. G. Douglas and Carl Pearcy, On a topology for invariant subspaces, J. Functional Analysis 2(1968), 323-341. MR 38 \#1547.

3. Peter Rosenthal, A note on unicellular operators, Proc. Amer. Math. Soc. 19(1968), 505-506. MR 36 \# 5753.

4. Donald Sarason, The $H^{p}$ spaces of an annulus, Mem. Amer. Math. Soc. No. 56(1965). MR 32 \#6256.

School of Mathematical Sciences, Flinders University, Bedford Park, South Australia 5042 